\title{
Learning for resilience-based management: Generating hypotheses from a behavioral study
}

David J. Yu ${ }^{1,2,3,6, *}$, Hoon C. Shin ${ }^{3,5}$, Irene Pérez ${ }^{3}$, John M. Anderies ${ }^{3,4,5}$, Marco A. Janssen ${ }^{3,4}$

${ }^{1}$ Lyles School of Civil Engineering, Purdue University

${ }^{2}$ Department of Political Science, Purdue University

${ }^{3}$ Center for Behavior, Institutions, and the Environment, Arizona State University

${ }^{4}$ School of Sustainability, Arizona State University

${ }^{5}$ School of Human Evolution and Social Change, Arizona State University

${ }^{6}$ Center for the Environment, Purdue University

* Current address: Lyles School of Civil Engineering, Purdue University, 550 Stadium Mall Drive, West Lafayette, IN 47907-2051, USA. Tel.: +1 480359 8310; fax: +1 7654940395. E-mail address: davidyu@purdue.edu

\section{Acknowledgements}

All authors gratefully acknowledge financial support from the National Science Foundation, Grant number GEO-1115054. 
1 Learning for resilience-based management: Generating hypotheses from a 2 behavioral study

3

\begin{abstract}
Encouragement of learning is considered to be central to resilience of socialecological systems (SESs) to unknown and unforeseeable shocks. However, despite the consensus on the centrality of learning, little research has been done on the details of how learning should be encouraged to enhance adaptive capacity for resilience. This study contributes to bridging this research gap by examining the existing data from a behavioral experiment on SES that involves learning. We generate new hypotheses regarding how learning should be encouraged by comparing the learning processes of human-subject groups that participated in the experiment. Our findings suggest that under environmental stability, groups may be able to perform well without frequent outer-loop (or double-loop) learning. They can still succeed as long as they tightly coordinate on shared strategies along with active monitoring of SESs and user participation in decision-making. However, such groups may be fragile under environmental variability. Only the groups that experience active outer-loop learning and monitoring of SESs are likely to remain resilient under environmental variability.
\end{abstract}

Key Words: loop learning; general resilience; behavioral experiment; adaptive management; adaptive co-management; adaptive governance.

\title{
1. Introduction
}

The dynamics of social-ecological systems (SESs) under global change are not well understood and are thus unpredictable (Polasky et al. 2011). Building the capacity to cope with unknown and unforeseeable shocks, or so-called general resilience, therefore requires continuous learning by actors to update the knowledge that they possess regarding the SESs they inhabit. Learning contributes to resilience via its positive influence on decision-making under uncertainty (Polasky et al. 2011, Biggs et al. 2015). The recognition of the importance of learning has given rise to several learning-focused management approaches that have garnered considerable interest over the years, such as adaptive management (Walters and Holling 1990), adaptive co-management (Olsson et al. 2004, Plummer and Armitage 2007), adaptive governance (Folke et al. 2005), and resilience engineering (Hollnagel et al. 2006). These approaches all embrace learning as a means to operationalize resilience-based management.

However, despite the consensus on the centrality of learning, little research has been done on the details of how learning should be encouraged to enhance adaptive capacity for resilience (Fabricius and Cundill 2014). Several studies have highlighted the issue as an important research gap, pointing out that the evidence supporting learning in the resilience literature does not indicate what type of learning is most appropriate and under what conditions (Biggs et al. 2012, 2015). This puzzle has arisen because a host of different learning-related conditions can interact to shape the outcomes of learning. For example, different types of learning can be present, such as multiple-loop learning (Argyris and Schön 1978, Keen et al. 2005) and individual and social learning (Reed et al. 2010). Learning can also be influenced by conditions such as the presence of diverse user participation in decision-making (Armitage et al. 2009), monitoring of and reflection on changes in SESs (Armitage et al. 2008), leadership and collaboration in the 
management process (Folke et al. 2005), and exchange of knowledge (Berkes 2009). Which combination of such conditions contributes to resilience the most? More effective strategies for building resilience can be identified by exploring how such conditions combine to shape outcomes and which of the configurations are more essential to adaptive capacity than others. The current study tackles this question by conducting a comparative analysis of the empirical data from a behavioral experiment on SES that involves learning. By comparing the learning processes of human-subject groups that participated in the experiment, we search for empirical clues and generate hypotheses regarding how learning should be encouraged for resilience-based management.

Our comparative analysis is based on an existing laboratory behavioral experiment of an irrigated agricultural system (Anderies et al. 2013a). In this experiment, which is designed to study how humans solve collective action problems under disturbances, participants are faced with a set of decision problems on collective management of shared irrigation infrastructure under environmental variability. Such behavioral experiments are typically used to test a precise hypothesis under a controlled setting (Poteete et al. 2010). Our approach of applying comparative analysis to existing experimental data to generate, rather than to test, hypotheses is an unusual research path to take, but is not without precedent (see Pavitt 2011, Perez et al. 2015). In essence, our approach reflects the methodological challenge raised by Basurto and Ostrom (2009) to advance theory development in the study of SESs, i.e., comparing rich details across multiple cases and combining theories to generate new hypotheses.

We examined the learning process of the experimental groups in terms of the following conditions: types of loop learning, user participation in decision-making, monitoring of and reflection on changes in SESs, group coordination in decision-making, sharing of knowledge, and initial level of knowledge from individual learning. Various configurations of the conditions emerged among the groups. For example, members of some groups coordinated on shared strategies with little loop learning while members of other groups often experienced loop learning. Of special interest is whether there is a configuration of conditions linked to successful group performance under environmental stability but which might be linked to failure under environmental variability. Understanding such a configuration can suggest hidden fragilities of seemingly well-functioning SESs that are typically only revealed through failure.

This paper first provides a theoretical background on how the learning process unfolds in SESs, then explains why our irrigation experiment likely offers empirical clues to understanding the details of learning processes linked to resilience. In the Methods section, we provide a brief overview of the design of the irrigation experiment, the protocol used to measure the learningrelated conditions, and the analytical approach used for comparative analysis. The remaining sections proceed through two stages of analysis. First, we analyzed which configurations of the conditions are likely to be linked to successful group performance under environmental stability. Second, we explored which configurations are likely to be linked to the resilience of that performance under environmental variability. Our findings suggest that resilient groups are characterized by frequent outer-loop learning (learning that involves revisions of shared goals or assumptions) and monitoring of and reflection on changes in SESs. We hypothesize that social capacity to learn and revise shared goals or assumptions in a flexible way through monitoring and evaluation is necessary for enhancing the general resilience of SESs. 


\subsection{Feedback-driven learning processes in SESs}

95

A group of actors typically gain knowledge about the SES they inhabit through the feedback loop processes shown in Figure 1. These processes involve iterations of monitoring of changes or outcomes in the SES, assessment of the monitored information leading to updated knowledge, and adjusting of subsequent management actions (Pahl-Wostl 2009). Such feedback-driven learning, or so-called loop learning, can occur at two levels: inner-loop (or single-loop) and outer-loop (or double-loop) (Argyris and Schön 1978, Keen et al. 2005). Inner-loop learning is defined as learning that involves updating of specific practices or actions to better meet existing goals or assumptions. This learning focuses on the question: Are we doing things right? Outerloop learning entails changes in shared goals or assumptions. This type of learning concerns the question: Are we doing the right things? Note that there is another higher level of loop learning (triple-loop learning) which involves changes in deep-seated beliefs and mental models (PahlWostl 2009). Triple-loop learning is not considered in this study because of the ambiguity associated with interpreting people's values and beliefs. Learning can also be either individual or social depending on how the learning takes place (Armitage et al. 2008, Newig et al. 2010). When knowledge is obtained by an individual and not by a collective group, individual learning is present. In contrast, social learning is present when learning goes beyond individuals and is situated within wider social units through social interactions (Reed et al. 2010, Rodela 2011).

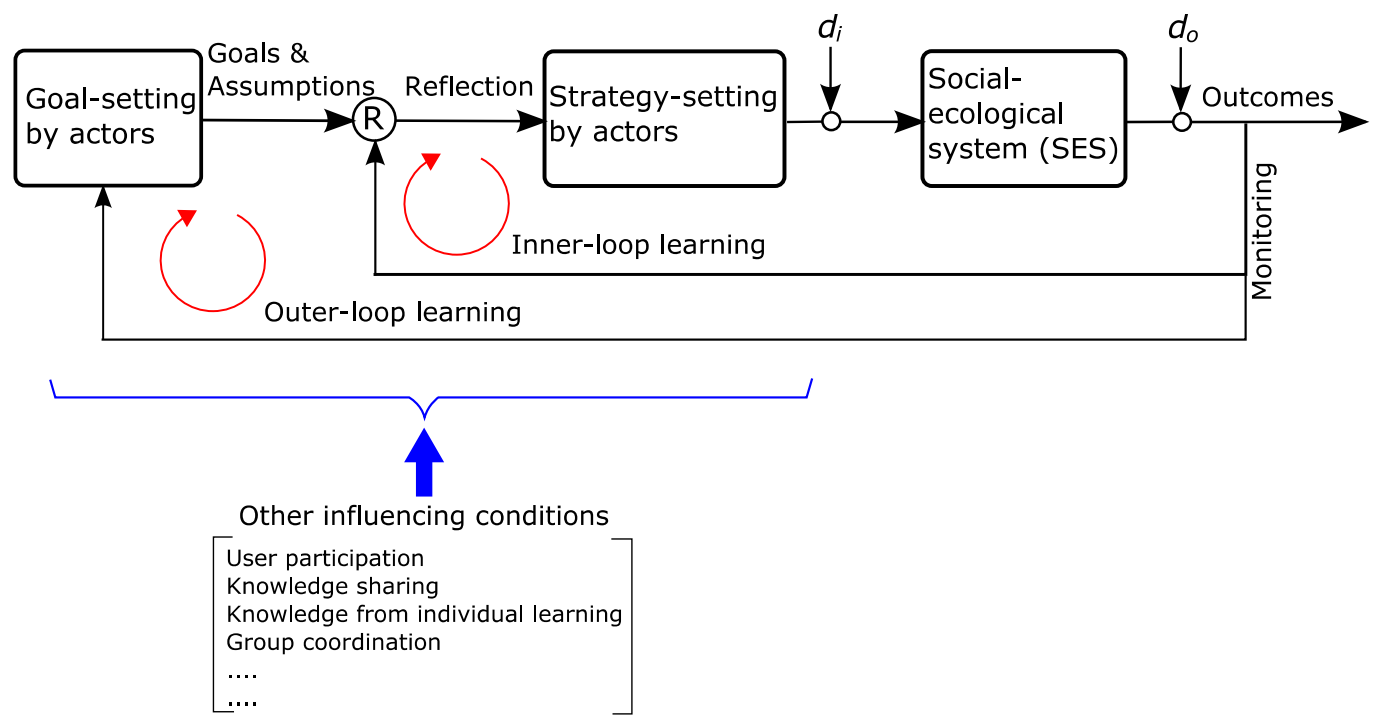

Figure 1. A conceptual diagram of how feedback-driven learning occurs in SESs. The inner-loop (or single-loop) learning entails fine-tuning of specific strategies or actions to better meet existing goals or assumptions. The outer-loop (or double-loop) learning involves updating of goals or assumptions that underlie specific strategies. The circle with letter $\mathrm{R}$ represents the process of monitoring of and reflection on past outcomes. The arrow denoted by $d_{i}$ represents internal issues (e.g., collective action problems). Environmental variability is represented by the arrows denoted by $d_{o}$ (e.g., natural disasters). Several conditions, e.g., user participation in decision-making, knowledge sharing, etc., can influence the loop learning processes. This figure is adapted from Anderies (2015) 
Several conditions can influence the loop learning processes (Fig. 1). In the context of our irrigation experiment, some of these conditions are the degree of user participation in decisionmaking, exchange of knowledge, monitoring of and reflection on changes in SES, and group coordination for management actions. User participation, defined as the process whereby diverse stakeholders actively engage in decision-making, is considered central to the co-management of SESs (Olsson et al. 2004). Broader user participation can facilitate pooling of knowledge from diverse stakeholders and thus likely has a positive influence on decision-making (Pahl-Wostl 2009, Djalante et al. 2011). Sharing of and access to knowledge regarding SESs can reinforce social memory and so influence how effectively groups adapt in the face of change and disturbance (Folke et al. 2007, Berkes 2009). Active exchange of knowledge can also stimulate group learning. Monitoring and reflection, defined as sensing and evaluation of changes or outcomes in a SES, is central to adaptive management because it connects learning and doing in a reciprocal manner (Fabricius and Cundill 2014). Effective ongoing monitoring and reflection likely affects how well SESs adapt under change.

Finally, how well individuals coordinate their actions as a group represents the level of collaboration or social cohesion in that group. Tight group coordination for management action can contribute to resilience in the form of resistance to change because it positively influences how efficiently a group responds to change. However, group coordination may also generate an unintended negative externality (Aldrich 2012). In the context of our irrigation experiment, this externality can be the dominance of a single group member or the blind conformity of group actions to one member's opinion, which ultimately undermines the group's capacity to innovate and change. When variability enters the SES and the complexity of the tasks escalates, this form of efficiency can quickly become a source of fragility. A related notion is the trap of social rigidity from traditions of understanding (Russell and Ison 2000). Social groups can become limited in their thinking and action because of their own historic legacies and business-as-usual mental models. Such traditions of understanding can lead to failures when social groups face new and unfamiliar circumstances. Hence, a related question that we explore in this study is whether or not tight group coordination can undermine adaptive capacity to change and surprise.

\subsection{Congruence between the irrigation experiment and feedback-driven learning}

The irrigation experiment is structured in such a way as to facilitate feedback-driven learning and institutional adaptation. In a SES in which humans maintain infrastructure to sustain an output, feedback typically occurs through the following sequence of steps. Actors sense the information about the system (e.g., changes in system outputs, individual behavior, biophysical conditions, etc.), translate this information into a set of social arrangements (e.g., shared strategies or goals), and then feed these arrangements back into the system to control the subsequent outputs or behaviors (Anderies et al. 2013b). The action situation of the irrigation experiment closely mirrors the sequence of steps above. In every round of the experiment, group members engage in group communication to assess outcomes of previous rounds and discuss how they should respond, to make decisions on the core problems of irrigation system management (infrastructure maintenance and water distribution), and then to observe the outcomes of those decisions. Group members experienced this sequence of steps for 20 rounds during the experiment. These repetitive problem-solving activities most likely facilitated group members to learn by doing. Indeed, repetitive problem-solving activities based on group communication and interactions 
171 with one another are known to reinforce shared knowledge and group learning (Ostrom 1992).

172 As such, a close examination of the experimental data will likely offer important clues for

173 identifying how learning should be encouraged to sustain a SES.

The experiment is also amenable to examining the details of the learning process linked to resilience because of the presence of environmental variability in selected rounds. In the first half of the experiment (rounds 1 to 10), group members solve the decision problems under environmental stability; i.e., the rate of the water supply feeding the irrigation infrastructure and the annual decline rate of the infrastructure are fixed. However, in the second half (rounds 11 to 20), they face the same decision problems under environmental variability; i.e., one of the two biophysical conditions above fluctuates unpredictably. If the performance of a group (tokens earned from growing crops using irrigated water) is maintained through both the stable and unstable rounds (Fig. 2A), we can assume that the SES is resilient. But if the performance decreases considerably under the unstable rounds, e.g., by more than a 50\% reduction (Fig. 2B), the system may be lacking in resilience.
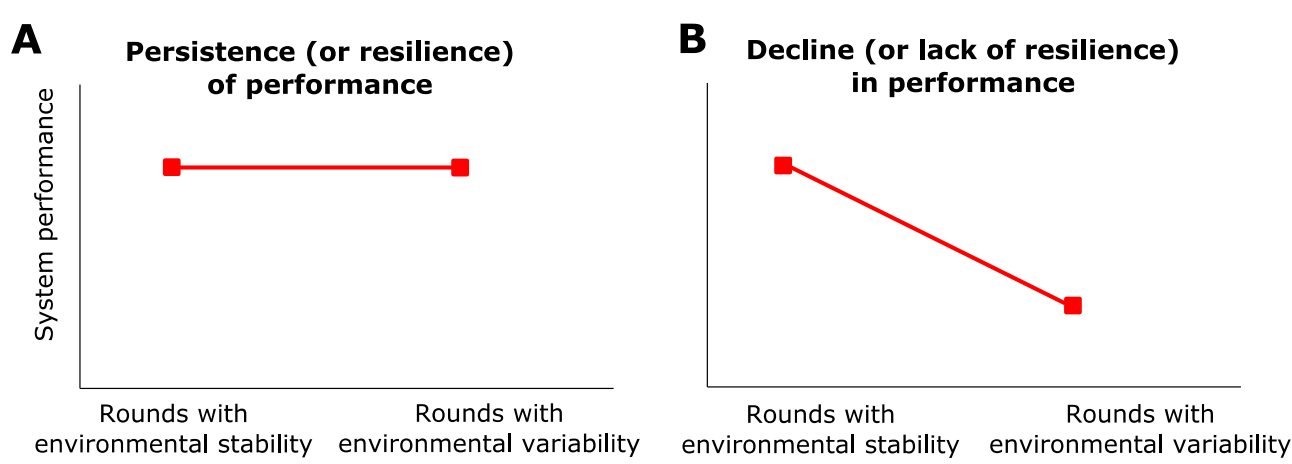

Figure 2. An illustration of resilience in the experimental irrigation system. The performance of the irrigation system can be expressed in terms of amount of tokens earned by a group from growing crops using irrigated water. Panel A illustrates persistence or resilience of the performance under environmental variability, i.e., tokens earned remain more or less consistent through both stable and unstable rounds. Panel B illustrates a considerable decline in the performance (thus lack of resilience) under environmental variability, i.e., tokens earned are significantly less under unstable rounds.

The irrigation experiment is designed to capture the essence of collective action problems associated with maintaining a farmer-managed irrigation system in a developing country context. In a typical setting, farmers produce crops by bringing water through production infrastructure (water diversion structure) and distribution infrastructure (canals). But this built environment poses two types of decision problems for farmers: the provision problem of shared infrastructure and the appropriation problem of irrigation water (Ostrom and Gardner 1993). First, farmers must mobilize a substantial level of collective labor to repair the infrastructure each year (canals must be cleaned of debris, and damaged water diversion structures must be repaired). If too few farmers participate in the endeavor, the performance of the irrigation system will decline and everyone loses (a threshold provision problem). Second, farmers must coordinate to receive a fair distribution of water, which can be difficult under the presence of upstream-downstream asymmetry (an asymmetric appropriation problem). Too much water collection by upstream farmers can subtract from the water that is available to downstream farmers. When this happens, 
downstream farmers who do not get enough water often retaliate by not contributing labor to the infrastructure maintenance in subsequent stages (Ostrom and Gardner 1993, Janssen et al. 2011).

\section{Methods}

This section provides a brief overview of the experimental design, the variables measured, and the analytical approach used. See Appendices A and B for more details on the experimental design and the protocol used for measuring the variables, respectively.

\subsection{Experimental action situation}

The irrigation experiment was conducted at Arizona State University in 2010. A randomlyrecruited sample of 105 undergraduate students (21 groups of 5 participants each) from various majors participated in the experiment. Upon arriving at the laboratory, participants were randomly seated at computer terminals separated by dividers. Before the experiment started, the nature of the tasks and payment procedure were explained using examples and two practice rounds. Participants' initial level of knowledge was tested with a short quiz.

Each group played 20 rounds of the experiment. A single round is comprised of the following three steps. In the first step, group members used a computer chat interface to communicate with each other for 60 seconds. Group members typically use this group chat to assess outcomes of previous rounds, devise social arrangements, and resolve conflicts regarding infrastructure maintenance and water distribution.

In the second step, each group member decides how much of his or her endowed asset (10 tokens each) to invest in maintaining infrastructure efficiency. Infrastructure efficiency can range from 0 to $100 \%$, with water delivery capacity being maximized at $100 \%$. Under stable conditions, it is optimal to maintain the infrastructure at $66 \%$ efficiency (see Fig. A1 in Appendix A). This investment decision involves a threshold public good problem because at least three group members need to make substantial investments to maintain the infrastructure efficiency (25 tokens are needed to offset the decline of $25 \%$ in the infrastructure efficiency in every round with stability). The resulting benefit of the investment, however, is open to all members regardless of their investment levels. Thus, self-interested rational actors would attempt to free-ride by taking water without investment.

In the third step, group members collect water over a period of 50 seconds to earn tokens from growing crops (see Fig. A1 in Appendix A). Five members in a group (denoted as positions A, B, $\mathrm{C}, \mathrm{D}$, and $\mathrm{E}$ in Fig. A2 in Appendix A) have heterogeneous access to water. Player A can access water before the others, while player $\mathrm{E}$ is the last to access water. This heterogeneity leads to the so-called asymmetric commons dilemma; i.e., the benefit is available to all, but individuals are heterogeneous in their capacity to obtain the benefit. In such a setting, advantaged individuals are tempted to take more benefit than the others, which subtracts from what is available to the others. Thus, self-interested rational players with prior access would take as much water as they can and leave little or no water for the others. 
Each group played rounds 1 to 10 under environmental stability; i.e., the rate of the water supply from the river was fixed at 30cf/s (cubic feet per second) and the infrastructure efficiency declined predictably by $25 \%$ per round. In rounds 11 to 20 , however, each group faced one of four treatments of environmental variability. The first two treatments related to variability in the infrastructure decline rate (Fig. 3A). In the infrastructure high variability (I-HV) treatment, the decline rate fluctuated between $10 \%$ and $80 \%$ over rounds 11 to 20 . In the infrastructure low variability (I-LV) treatment, the decline rate fluctuated between $15 \%$ and $35 \%$. A high decline rate, e.g., $80 \%$, mimics a severe flashflood that significantly damages the irrigation infrastructure. The latter two treatments related to variability in the rate of water supply from rivers feeding the infrastructure (Fig. 3B). In the water supply high variability treatment (W-HV), the supply rate fluctuated between $20 \mathrm{cf} / \mathrm{s}$ and $40 \mathrm{cf} / \mathrm{s}$ over rounds 11 to 20 . This variability was reduced to the range of $25 \mathrm{cf} / \mathrm{s}$ and $35 \mathrm{cf} / \mathrm{s}$ under the water supply low variability (W-LV) treatment. A low supply rate, e.g., $20 \mathrm{cf} / \mathrm{s}$, represents a drought or reduced water flow in the river. Note that the infrastructure efficiency of each group was initialized to $75 \%$ at the start of rounds 1 and 11 . For more details on the experimental design, see Appendix A.
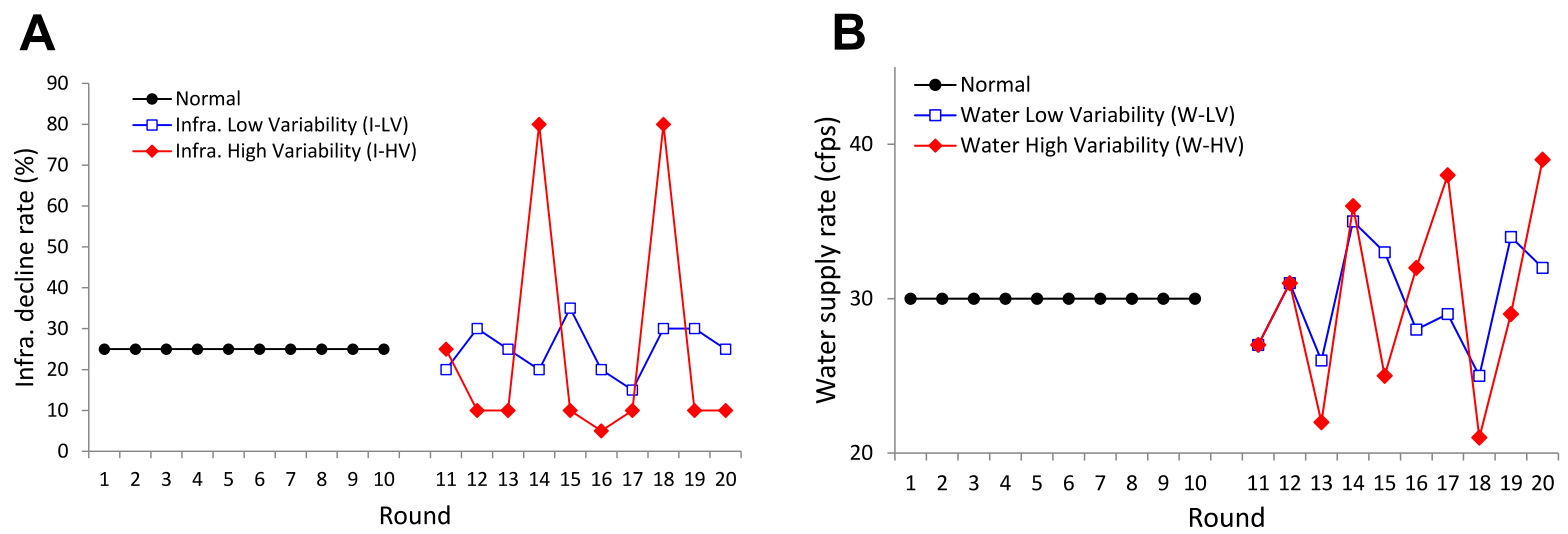

Figure 3. Four treatments of environmental variability are introduced in rounds 11 to 20. Panel A shows high and low variability in the infrastructure decline rate. Panel B shows high and low variability in the water supply rate. The tally of the groups that played the four treatments are five groups for I-LV (groups 1 to 5), six groups for I-HV (groups 6 to 11), five groups for W-LV (groups 12 to 16), and five groups for W-HV treatments (groups 17 to 21). This figure is adapted from Anderies et al. (2013a).

\subsection{Outcome variables: Performance and resilience}

Two outcome variables are measured in this study: group performance in terms of tokens earned from growing crops in rounds 6 to 10 (stable rounds) and the resilience of that performance through rounds 11 to 15 (unstable rounds, Table 1). We only considered rounds 6 to 10 in measuring the performance under environmental stability because players typically take a few early rounds to stabilize their behavior. While the low crop production in rounds 1 to 5 may be of a transient nature, the same phenomenon in rounds 6 to 10 is a clearer sign of poor group performance.

The resilience of group performance under environmental variability is assessed by comparing tokens earned from growing crops between the stable and unstable rounds. We derived the ratio 
of tokens earned from growing crops in rounds 11 to 15 to those in rounds 6 to 10 . We considered rounds 11 to 15 for the unstable rounds because they more clearly represented the initial-stage behaviors of the groups in response to the shocks compared to in rounds 16 to 20 . Ratio values of around 1.0 indicated little or no change in group performance despite the below 1.0 (less than 0.5 ) clearly signified a large decline in group performance under variability (thus, a sign of lack of resilience). See Appendix B for the detailed criteria we used to measure the outcome variables.

Note that groups that performed poorly in rounds 6 to 10 but performed better or similarly in rounds 11 to 15 are likely to have a ratio value close to or above 1.0. Groups 5 and 12 belong to such groups. We excluded these groups in our analysis for resilience because their ratio values were misleading for interpreting resilience. Because of their failure in rounds 6 to 10 , the maintenance of their performances in rounds 11 to 15 has no meaning for understanding the persistence of good performance.

Note also that the infrastructure efficiency of every group was initialized to $75 \%$ at the start of the experiment (round 1) and just before the variability treatments were applied (round 11). Hence, the ratio derived by comparing rounds 11 to 15 and rounds 6 to 10 might be slightly altered because of the initialization done at round 11. To address this issue, we performed an alternate measurement of the resilience by deriving the ratio of tokens earned from growing crops in rounds 11 to 20 to those in rounds 1 to 10 . We checked the robustness of our comparative analysis results using this alternate measurement.

\subsection{Coding the causal conditions}

We considered seven conditions in characterizing the learning processes of the experimental groups. These conditions included different types of loop learning (inner- and outer-loop), user participation in the decision-making process, knowledge sharing, monitoring of and reflection on changes in SESs, group coordination on shared strategies, and initial level of knowledge from individual learning (Table 1). We measured these conditions by analyzing the content of the group communication and group members' decisions regarding infrastructure maintenance and water distribution. In this subsection, we explain how we measured loop learning. The protocol used to measure the remaining conditions is explained in Appendix B.

To measure loop learning, we need to clearly define two levels of social arrangements: 1) social goal or assumption and 2) group strategy. A social goal or assumption is a shared objective or a normative basis that guides group members' specific actions or practices. An example would be group members agreeing on fair distribution of water or optimal maintenance of infrastructure. Group strategy is a specific action or practice shared within a group that is devised to meet a social goal or assumption. An example would be group members agreeing on investing six tokens each to reach $80 \%$ infrastructure efficiency. Another example would be group members agreeing on rotating water collection for 10 seconds in order to achieve fair distribution of water. Note that, although the participants were given explanations about the working logic of the irrigation system in the pre-experiment stage, they were not told which social arrangements 
(shared goals or strategies) to follow. It was up to them to decide which social arrangements to adopt, if any, as they played the experiment.

With this definition in mind, we see that 1) the existence of a group strategy and 2) whether there is a change in that strategy is a sign of loop learning in action. If the majority of group members (three or more) follow a shared rule for either the investment or the water distribution in a given round, we assumed that a group strategy was present in that round. For example, a group strategy likely exists when three or more members invest the same amount of tokens or collect the same amount of water based on agreements made through group discussion. Whenever such a group strategy is revised in a given round to better meet an existing goal, we treated it as an occurrence of inner-loop learning. For example, if a shared goal was to collect water equally, group members would try various water distribution strategies and learn from those experiences to reach the desired goal. Likewise, if a shared goal was to maintain the infrastructure efficiency around $66 \%$, they would try various investment strategies and learn from those experiences to reach the goal.

Similarly, an outer-loop learning may be happening when a group strategy is revised to meet a new goal or assumption. For example, if the content of a group discussion reveals that members share a revised assumption in which $66 \%$ infrastructure efficiency is better than $81 \%$ efficiency and if their subsequent investment patterns reflect this new goal, an outer-loop learning probably happened. See Appendix B for more discussion on the protocol used to measure loop learning as well as the strengths and limitations of this approach.

The remaining conditions (user participation in the decision-making process, knowledge sharing, monitoring and reflection, group coordination, and initial level of knowledge from individual learning) were measured using the coding rules outlined in Appendix B. Using this set of coding protocols, two of the authors independently measured the seven conditions. After the measurements, the two authors compared their coding results and reached a consensus through discussion on their re-assessment of the conditions. The inter-coder reliability of this coding effort turned out to be acceptable. For more details on the reliability of our coding effort, see Appendix B.

Table 1. Definitions of the outcome variables and the learning-related conditions that may affect the resilience of SES.

\begin{tabular}{ll}
\hline \hline Variables & Definitions \\
\hline $\begin{array}{l}\text { Outcome variables: } \\
\text { Group performance }\end{array}$ & $\begin{array}{l}\text { The level of group tokens earned from growing crops using } \\
\text { irrigated water in rounds } 6 \text { to } 10 \text { (stable rounds). The amount of } \\
\text { tokens earned by a group from growing crops in rounds } 6 \text { to } 10 \text { is } \\
\text { calibrated }{ }^{\dagger} \text { using the standard QCA software tool. The resulting } \\
\text { measure has a continuous scale of } 0-1.0 \text { (clearly low } \\
\text { performance=0, clearly high performance=1.0). }\end{array}$ \\
$\begin{array}{l}\text { The level of continuity in group performance under environmental } \\
\text { pesilience of group } \\
\text { (RESIL) }\end{array}$ & $\begin{array}{l}\text { gariability. This measure is derived by calculating the ratio of } \\
\text { (unstable rounds) to those in rounds } 6 \text { to } 10 . \text { The resulting measure }\end{array}$
\end{tabular}


Causal conditions: ${ }^{\S}$ Inner-loop learning (INNER)

Outer-loop learning (OUTER)

User participation (UPART)

Knowledge-sharing (KNOW)

Group coordination (COORD)

Monitoring and reflection

(MOREF)

Knowledge from individual learning (QUIZ) is then calibrated ${ }^{\ddagger}$ to have a continuous scale of $0-1.0$ (clearly fragile $=0$, clearly resilient $=1.0$ ).

How often group strategies for either infrastructure investment or water collection decisions are revised to meet an existing goal or assumption. This measure has a discrete scale of four levels (clearly often $=1.0$, more often than not $=0.67$, seldom $=0.33$, clearly never $=0.0$ ).

How often goals or assumptions regarding infrastructure investment or water collection decisions are revised. This measure has a discrete scale of four levels (clearly often $=1.0$, more often than not $=0.67$, seldom $=0.33$, clearly never $=0.0$ ).

The level of user participation in the decision-making process for group strategies. This measure has a discrete scale of four levels (clearly often $=1.0$, more often than not $=0.67$, seldom $=0.33$, clearly never $=0.0$ ).

The level of information exchange regarding the knowledge of the experimental action situation. This measure has a discrete scale of four levels (clearly often $=1.0$, more often than not $=0.67$, seldom $=0.33$, clearly never $=0.0$ ).

The level of coordination among participants for group strategies in both the investment and water collection decisions. This measure has a discrete scale of four levels (clearly often $=1.0$, more often than not $=0.67$, seldom $=0.33$, clearly never $=0.0$ ).

The level of monitoring and reflection regarding (1) group- or individual-level outcomes, (2) conditions of biophysical components, or (3) correcting of non-conforming behaviors. This measure has a discrete scale of four levels (clearly often $=1.0$, more often than not $=0.67$, seldom $=0.33$, clearly never $=0.0$ ).

The initial level of knowledge on how the irrigation system works obtained through individual learning. Before the experiment started, individuals were given explanations about the working logic of the irrigation system, and their knowledge was measured using a short quiz. This variable is based on the average score of the quiz taken by group members. The variable is expressed in a discrete scale of four levels (excellent understanding=1.0, good understanding $=0.67$, some understanding $=0.33$, poor understanding $=0.0)$.

†, The standard QCA software tool (fs/QCA) developed by Charles Ragin (2008) was used to conduct the calibration. For more details on the criteria we used for calibration, see Appendix B. $\S$ The measurements of INNER, OUTER, UPART, KNOW, COORD, are MOREF are based on the assessment of two coders who shared a same set of coding protocols. For more details, see Appendix B.

\subsection{Analytical approach}


We used fuzzy-set qualitative comparative analysis (fsQCA) to compare the learning processes of the experimental groups. Our objective was to find multiple causal configurations of the conditions linked to the two outcome variables. Given the middle range N sample size (21 groups) and our objective of addressing the issue of multi-causality, fsQCA was identified as the best analytical method available to us. fsQCA is based on Boolean algebra and treats an empirical case as a logical configuration of set-memberships in different conditions and outcomes (Ragin 1987, 2000). Researchers can use fsQCA to identify all possible configurations of conditions that may be causally associated with an outcome of interest (Basurto 2013). The set-theoretic assumption allows researchers to establish the conditions of necessity and sufficiency (Ragin 2008). A condition is necessary if it appears in all configurations tied to an outcome. A condition is sufficient but not necessary if its presence is associated with an outcome in a certain configuration but is not the only condition with that association; i.e., there is another condition in a different configuration associated with that outcome. A condition is both necessary and sufficient if it is tied to an outcome and is the only condition with that linkage. See Ragin $(2000,2008)$ for more details on the method. Several exemplary applications of fsQCA exist in

Our analysis proceeds through two stages. First, we conducted fsQCA to identify potential causal configurations of the conditions linked to group performance under environmental stability. fs/QCA, the standard QCA software tool developed by Charles Ragin (2008), was used for the analysis. Second, we used fs/QCA to identify which configurations are likely to be causally linked to the resilience of group performance under environmental variability, i.e., comparing group performance in rounds 11 to 15 to that in rounds 6 to 10 . Two sub-samples of the data were used in the second stage: groups with high or low variability treatments and groups with only high variability treatments. The first sub-sample allowed us to identify potential causal configurations linked to more general adaptive capacity, irrespective of the differences in the degree of variability. With the second sub-sample, we focused more on configurations linked to the capacity to cope with severe environmental shocks. If the results based on the two subsamples show a consistent pattern, we can have more confidence in the results obtained. As a robustness check, we also repeated the second analysis using an alternate measurement of the resilience, i.e., a comparison of group performance in rounds 11 to 20 to that in rounds 1 to 10 .

\section{Results}

This section provides the results of our comparative analysis, the causal configurations linked to the two outcome variables (Table 2). It also shows trends in the two outcome variables as shown in Figure 4.

\subsection{Trends in group performance and its resilience}

Figure 4 displays the change in the group performance between the stable rounds (rounds 6 to 10 ) and the unstable rounds (rounds 11 to 15 ). In the stable rounds, the mean and the median group earnings from crop production were 249 and 268 tokens, respectively, with a minimum earnings of 58 tokens and a maximum earnings of 301 tokens. Among the 21 groups, 16 groups earned at least 250 tokens in the stable rounds. Hence, most of the groups showed more or less similar performance, earning tokens close to the measures of the average. 
Note that groups 5 and 12 failed to perform well in the stable rounds (Fig. 4). Barring these two groups, the mean and the median percentage change in tokens earned between the stable and unstable rounds (a ratio of tokens earned in rounds 11 to 15 over those in rounds 6 to 10 subtracted by 1.0 ) were $-18 \%$ and $-14 \%$, respectively. The most severe decline in group performance was $-64 \%$ (group 13, W-LV treatment). The largest gain in group performance was $+6 \%$ (group 2, I-LV treatment). Figure 4 also reveals that the groups with high variability treatments (I-HV and W-HV) more consistently experienced a decline in group performance in the unstable rounds. The averages of percentage changes in group performance for the I-HV, I$\mathrm{LV}, \mathrm{W}-\mathrm{HV}$, and $\mathrm{W}-\mathrm{LV}$ treatment groups were $-24 \%,+1 \%,-28 \%$, and $-2 \%$, respectively. This pattern was expected because the high variability treatments caused more adversity in the affected groups. A cross-table comparison was also done between the two outcome variables and the learning-related conditions (see Table $\mathrm{C} 1$ in Appendix C).
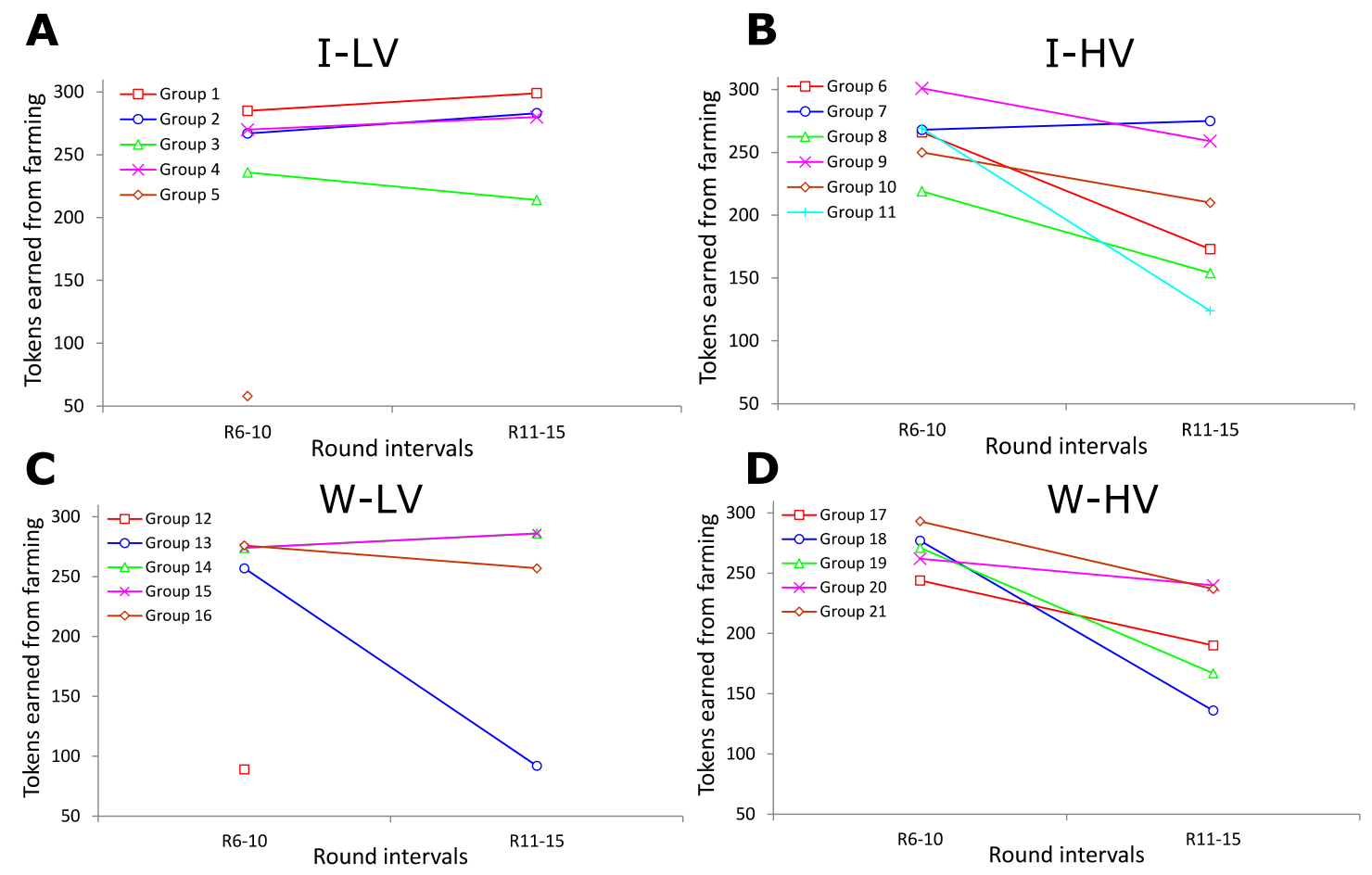

Figure 4. Trends in group performance and its resilience in the irrigation experiment. Panels A and B show the changes in group performance between rounds 6 to 10 (stable rounds) and rounds 11 to 15 (unstable rounds) for the infrastructure low variability (I-LV) and the infrastructure high variability (I-HV) treatment groups, respectively. Panels C and D show the changes in group performance between rounds 6 to 10 and rounds 11 to 15 for the water supply low variability (W-LV) and the water supply high variability (W-HV) treatment groups, respectively. Note that groups 5 and 12 performed poorly in rounds 6 to 10, and are thus excluded from our analysis.

\subsection{Causal configurations for performance}

Our fsQCA results suggest two causal configurations linked to group performance under 450 environmental stability. These configurations are (C1) "group coordination" combined with "user 
participation" and "monitoring and reflection" and (C2) "inner-loop learning" combined with "outer-loop learning" and "monitoring and reflection" (Table 2). The presence of "monitoring and reflection" in both causal configurations suggests that this is a necessary condition for the outcome. $\mathrm{C} 1$ signifies that if group members frequently exhibit diverse participation in decisionmaking and follow shared strategies while regularly evaluating past outcomes, they can achieve good performance. C2 suggests that if individuals frequently experience both inner- and outerloop learning while regularly evaluating past outcomes, they can also achieve good performance. configurations. For more details on the fsQCA results, see Appendix C.

\subsection{Causal configurations for resilient performance}

The fsQCA results also show multiple causal configurations for the resilience of group performance. We identified two causal configurations based on the high or low variability treatment groups: (C3) "inner-loop learning" combined with "outer-loop learning" and "monitoring and reflection" and (C4) "group coordination" combined with "outer-loop learning," "user participation," and "monitoring and reflection" (Table 2). Note that C1 and C3 are identical. C2 and C4 differ by only one condition; i.e., C4 has set-membership to "outer-loop learning" while $\mathrm{C} 2$ does not. The condition of "monitoring and reflection" again appears in all of the configurations. An especially noteworthy result is that all of the configurations now include the condition of "outer-loop learning."

The same analysis based on the groups with high variability treatments reveals four causal configurations (C5 to C8; Table 2). Note that these four configurations are almost indistinct; i.e., they have set-memberships to almost all of the conditions with only slight variations.

Nonetheless, we can make two observations. First, the four configurations (C5 to C8) have setmemberships to "outer-loop learning" and "monitoring and reflection." This regularity reinforces our earlier interpretation of C3 and C4. Second, the condition of "knowledge from individual learning" finally appears in some of the configurations (C6 and C7), though one configuration (C8) has set-membership to the complement of that condition. The condition, "knowledge from individual learning," is still neither necessary nor sufficient for the outcome. "Knowledge sharing" also appears in some of the conditions (C6 to C8). For more details on the fuzzy-set truth table construction and the consistency and coverage scores of the logical configurations, see Appendix C.

We also did a robustness check of the causal configurations identified above based on an alternate measurement of resilience, i.e., a comparison of group performance in rounds 11 to 20 to that in rounds 1 to 10 . The fsQCA results based on the alternate measurement reveal the same pattern: The conditions of "outer-loop learning" and "monitoring and reflection" appear in all of the causal configurations (C9 to C13 in Table C11 in Appendix C). This consistency reinforces our observation that the two conditions are necessary for resilient performance.

Table 2. Comparison of the causal configurations linked to group performance (PERFORM) under environmental stability and the resilience of group performance (RESIL) under environmental variability. The causal conditions considered are inner-loop learning (INNER), outer-loop learning (OUTER), user participation (UPART), knowledge-sharing (KNOW), group 
coordination (COORD), monitoring and reflection (MOREF), and knowledge from individual learning (QUIZ). For more details on the fsQCA outputs, see Appendix C.

\begin{tabular}{|c|c|c|}
\hline \multirow{2}{*}{$\begin{array}{l}\text { PERFORM } \\
(\mathrm{N}=21)\end{array}$} & \multicolumn{2}{|c|}{ RESIL } \\
\hline & $\begin{array}{l}\text { Low and high variability } \\
\qquad(\mathrm{N}=19)\end{array}$ & $\begin{array}{l}\text { High variability } \\
\qquad(\mathrm{N}=11)\end{array}$ \\
\hline Three configurations: & Two configurations: & Four configurations \\
\hline $\begin{array}{l}\text { (C1) INNER and OUTER and } \\
\text { MOREF }\end{array}$ & $\begin{array}{l}\text { (C3) INNER and OUTER and } \\
\text { MOREF }\end{array}$ & $\begin{array}{l}\text { (C5) COORD and INNER and } \\
\text { OUTER and UPART and } \\
\text { MOREF }\end{array}$ \\
\hline $\begin{array}{l}\text { OR } \\
(\mathrm{C} 2) \mathrm{COORD} \text { and UPART } \\
\text { and MOREF }\end{array}$ & $\begin{array}{l}\text { OR } \\
\text { (C4) COORD and UPART } \\
\text { and MOREF and OUTER }\end{array}$ & $\begin{array}{l}\text { OR } \\
\text { (C6) INNER and OUTER and } \\
\text { UPART and KNOW and } \\
\text { MOREF and QUIZ } \\
\text { OR } \\
\text { (C7) COORD and INNER and } \\
\text { OUTER and KNOW and } \\
\text { MOREF and QUIZ } \\
\text { OR } \\
\text { (C8) COORD and OUTER and } \\
\text { UPART and KNOW and } \\
\text { MOREF and not QUIZ }\end{array}$ \\
\hline
\end{tabular}

\section{Discussion and conclusions}

A growing body of literature indicates that learning is essential to building the capacity of actors in SESs to deal with all kinds of shocks, including novel ones. For example, it has been recently suggested that encouragement of learning is one of seven key principles for enhancing the resilience of ecosystem services in the face of disturbances and change in SESs (Biggs et al. 2015). Learning contributes to resilience via its positive influence on decision-making under uncertainty (Polasky et al. 2011, Biggs et al. 2015).

However, despite the consensus on the centrality of learning, little research has been done to critically analyze the details of the learning process linked to adaptive capacity for resilience (Fabricius and Cundill 2014). Several studies have underscored this issue as an important research gap, indicating that the evidence in support of learning in the resilience literature does not specify what type of learning is most appropriate and under what conditions (Biggs et al. 2012, 2015). This study contributes to bridging the research gap by examining empirical data from a behavioral experiment on SES that involves learning. Through an analysis of the experimental data, we generated new hypotheses regarding how learning should be encouraged for resilience. Several learning-related conditions were measured using the data (Table 1). Our objective was to find multiple causal configurations on the conditions linked to 1) group performance under environmental stability and 2) the resilience of group performance under environmental variability. 
523 Using fuzzy-set qualitative comparative analysis (fsQCA), we found two causal configurations

524 for group performance under environmental stability ( $\mathrm{C} 1$ and $\mathrm{C} 2$; Table 2). Two interesting

525 observations were made from this result: monitoring of and reflection on changes in SESs is a necessary condition for achieving good performance, and one configuration involves frequent loop learning (C2), while the other is based on group coordination and user participation in management $(\mathrm{C} 1)$. These observations suggest that there are two very different paths leading to the same outcome when the disturbance regime is predictable. In one path, group members seldom experiment with different social arrangements and thus experience little learning by doing. They adhere to some initially chosen goals or strategies and constrain non-conforming behaviors. Some members may suggest alternative social arrangements, but these proposals are rarely accepted because there is often a dominant opinion leader who directs the whole group. In the other path, group members show less group coordination, but they are willing to try different social arrangements. They actively monitor and reflect on the outcomes of these trials and learn

We found multiple causal configurations for the resilience of group performance under environmental variability: two configurations from all the variability treatments $(\mathrm{C} 3$ and $\mathrm{C} 4$; Table 2) and four configurations from the high variability treatments (C5 to C8; Table 2). All of these configurations have set-memberships to "outer-loop learning" in addition to "monitoring and reflection." This regularity implies that both conditions are necessary for the resilience of group performance under variability. C3 and C4 suggest that the mode of governance driven by group coordination alone is no longer effective when variability enters the system. C5 to C8 show indistinct patterns that are difficult to interpret, possibly because of the small size of the sub-sample $(\mathrm{N}=11)$ used for fsQCA. Nonetheless, all four configurations have set-memberships to "outer-loop learning" and "monitoring and reflection," and therefore reinforce our interpretation of $\mathrm{C} 3$ and $\mathrm{C} 4$. The criticality of "monitoring and reflection" is obvious. We often renew our knowledge and acquire best practices for complex tasks by constantly adjusting our decisions and monitoring and assessing the outcomes of those decisions (Stiglitz and Greenwald 2014). Monitoring and reflection facilitates the critical interplay between learning and doing. Without the presence of this condition, adaptive management of SES is impossible.

What we find especially interesting is the importance of outer-loop learning under environmental variability. When the disturbance regime is predictable (i.e., the infrastructure decline rate and water supply rate are fixed at $25 \%$ and $30 \mathrm{cfps}$, respectively), groups may still be able to perform well without frequent loop learning. As long as they tightly coordinate on shared strategies along with active monitoring and reflection and user participation, they can effectively resist the change and achieve good system performance. Our results suggest that such groups may be fragile when variability enters the system. This situation possibly represents the "dark side" or double-edged nature of tight group coordination with little loop learning. Although group coordination brings the benefit of efficient response, it may also inhibit a group's capacity to innovate and change when necessary.

The importance of outer-loop learning is also consistent with the notion of transformability of SES. Transformability, defined as the capacity of SES to change system structure and identity and patterns of interactions when an existing system becomes untenable (Walker et al. 2004, 568 Folke et al. 2010), likely requires renewal of social goals and assumptions shared by the actors in 
the system. This was perhaps the case when group members faced environmental variability. They needed to transform shared goals and assumptions that had been fine-tuned to a stable operating environment. Groups that had not developed enough transformability through frequent outer-loop learning probably could not easily break away from their conventional mode of governance or traditions of understanding when the shocks came. We conjecture that such groups missed the window of opportunity to transform their management approach because of their lack of experience with outer-loop learning.

Group 11 is an example of a group that performed well with little loop learning in the stable rounds but failed to continue that trend when variability (I-HV) entered the system. Our data show that members of group 11 rarely experimented with different target levels of infrastructure efficiency or water distribution norms in the stable rounds. Most group members faithfully followed shared social arrangements, which allowed them to achieve good performance until round 10. However, when the disturbance regime became unpredictable starting in round 11, they could not effectively adjust their behavior. A real world example of group 11 might be found in the nuclear catastrophe at the Fukushima Daiichi nuclear plant in Japan in 2011. Although the nuclear plant did survive the earthquake, planners had not foreseen the simultaneous effects of a tsunami and associated infrastructure failures. This unanticipated situation forced the planners to be highly creative and adaptive in their response. Unfortunately, the lack of such a response in the initial stages of the disaster exacerbated the catastrophe (Park et al. 2011). The rigidity of the planners' response might have been caused by their traditions of understanding and fixation with existing manuals of operation, which tend to be more prevalent in a hierarchical society such as Japan.

A prime example of a group that actively engaged in outer-loop learning is group 7. Despite facing severe environmental variability (I-HV), this group was able to perform well under both the stable and unstable rounds. At the outset, group members quickly tested the goals of maintaining the infrastructure near $100 \%$ efficiency and of equal sharing of water. In the subsequent rounds, they managed to revise the shared goals to the following: maintain infrastructure efficiency at a moderate level (around 81\%) and use water both equally and efficiently (each member should get an equal amount of water and player $\mathrm{E}$ must always leave his or her gate open to prevent water wastage). We speculate that this experience improved the group's capacity to innovate and change. This flexibility was indeed demonstrated in round 11 when the group members anticipated that a catastrophic infrastructure decline might be on the way and revised their investment target to $100 \%$ efficiency. They reasoned that $100 \%$ efficiency would create a larger cushion to counter a major decline in efficiency. Real world examples of group 7 might be represented by long-lived farmer-managed irrigation systems in Asia (e.g., Bacdayan 1974, Gupta and Tiwari 2002). These systems are distinguished by well-functioning collective choice arena that enable participants to deliberate and flexibly adapt their daily operational rules or policies under a changing environment (Ostrom 1990). The presence of wellfunctioning collective choice arena could be interpreted as a sign of active outer-loop learning.

Finally, it should be noted that none of the conditions are either sufficient or both necessary and sufficient. In particular, the results show that the conditions of initial level of knowledge from individual learning and exchange of knowledge are not necessary for good performance and its resilience. Recall that every experimental participant was given an explanation about the 
working logic of the irrigation system, and this knowledge was measured by a short quiz in the pre-experiment stage. Hence, the knowledge was obtained by an individual with imposition of information from the outside. Our findings therefore suggest the limitations of imposed forms of individual learning and mere exchange of knowledge. Learning by doing acquired at the group level through social interactions may be more essential for building adaptive capacity.

In summary, our findings indicate that active outer-loop learning and monitoring of and reflection on changes in SESs are key conditions for building general resilience of SESs. Policymakers interested in improving resilience in the form of adaptive capacity should thus promote the culture of the two conditions in their focal SESs. We also suggest some direction for future studies. Our research is based on a laboratory behavioral experiment with undergraduate students at a U.S. university as the participants. Future studies may consider conducting a similar experiment in a field setting with actual farmers as participants to incorporate more contextual considerations into the analysis. Further, it has been recently suggested that learning and innovation are elements of adaptive governance that have a high potential to contribute to the study of disaster risk reduction governance (Djalante et al. 2011). Designing and conducting a new behavioral experiment in the context of disaster management of a critical infrastructure may be an important next step in research on disaster risk reduction. Insights learned from different contexts and their comparisons, including ours, will contribute to a more complete understanding of how learning should be encouraged to promote the general resilience of human-dominated complex systems.

\section{Literature Cited}

Aldrich, D. P. 2012. Social, not physical, infrastructure: the critical role of civil society after the 1923 Tokyo earthquake. Disasters 36(3):398-419.

Anderies, J. M. 2015. Understanding the Dynamics of Sustainable Social-Ecological Systems: Human Behavior, Institutions, and Regulatory Feedback Networks. Bulletin of Mathematical Biology 77(2):259-280.

Anderies, J. M., M. A. Janssen, Lee, Allen, and H. Wasserman. 2013a. Environmental variability and collective action: Experimental insights from an irrigation game. Ecological Economics 93:166-176.

Anderies, J. M., E. Ostrom, C. Folke, and B. Walker. 2013b. Aligning Key Concepts for Global Change Policy: Robustness, Resilience, and Sustainability. Ecology and Society 18(2):8.

Argyris, C., and D. Schön. 1978. Organizational Learning. Addison-Wesley, Reading, MA.

Armitage, D., M. Marschke, and R. Plummer. 2008. Adaptive co-management and the paradox of learning. Global Environmental Change 18:86-98.

Armitage, D. R., R. Plummer, F. Berkes, R. I. Arthur, A. T. Charles, I. J. Davidson-Hunt, A. P. Diduck, N. C. Doubleday, D. S. Johnson, M. Marschke, P. McConney, E. W. Pinkerton, and E. K. Wollenberg. 2009. Adaptive co-management for social-ecological complexity. Frontiers in Ecology and the Environment 7(2):95-102.

Bacdayan, A. 1974. Securing water for drying rice terraces: irrigation, community organization, and expanding social relationships in a Western Bontoc group, Philippines. Ethnology 13(3):247-260. 
701

702

703

704

Basurto, X. 2013. Linking multi-level governance to local common-pool resource theory using fuzzy-set qualitative comparative analysis: Insights from twenty years of biodiversity conservation in Costa Rica. Global Environmental Change 23(3):573-587.

Basurto, X., and E. Ostrom. 2009. The Core Challenges of Moving Beyond Garrett Hardin. Journal of Natural Resources Policy Research 1(3):255-259.

Berkes, F. 2009. Evolution of co-management: role of knowledge generation, bridging organizations and social learning. Journal of environmental management 90(5):1692-702.

Biggs, R., M. Schlüter, D. Biggs, E. L. Bohensky, S. BurnSilver, G. Cundill, V. Dakos, T. M. Daw, L. S. Evans, K. Kotschy, A. M. Leitch, C. Meek, A. Quinlan, C. Raudsepp-Hearne, M. D. Robards, M. L. Schoon, L. Schultz, and P. C. West. 2012. Toward Principles for Enhancing the Resilience of Ecosystem Services. Annual Review of Environment and Resources 37(1):421-448.

Biggs, R., M. Schlüter, and M. L. Schoon. 2015. Principles for Building Resilience: Sustaining Ecosystem Services in Social-Ecological Systems. Cambridge University Press, Cambridge, UK.

Djalante, R., C. Holley, and F. Thomalla. 2011. Adaptive governance and managing resilience to natural hazards. International Journal of Disaster Risk Science 2(4):1-14.

Fabricius, C., and G. Cundill. 2014. Learning in adaptive management: insights from public practice. Ecology and Society 19(1):29.

Folke, C., S. R. Carpenter, B. Walker, M. Scheffer, and T. Chapin. 2010. Resilience Thinking: Integrating Resilience, Adaptability and Transformability. Ecology And Society 15(4):20.

Folke, C., T. Hahn, P. Olsson, and J. Norberg. 2005. Adaptive Governance of Social-Ecological Systems. Annual Review of Environment and Resources 30(1):441-473.

Folke, C., L. Pritchard, F. Berkes, J. Colding, and U. Svedin. 2007. The Problem of Fit between Ecosystems and Institutions : Ten Years Later. Ecology And Society 12(1):30.

Gupta, R., and S. Tiwari. 2002. At the crossroads: continuity and change in the traditional irrigation practices of Ladakh. Paper presented at the Commons in an Age of globalization, Nineth conference of International Association for the Study of Common Property. Victoria Falls, Zimbabwe.

Hollnagel, E., D. Woods, and N. Leveson. 2006. Resilience engineering: Concepts and precepts. Ashgate, Aldershot, UK.

Janssen, M. A., J. M. Anderies, and J. C. Cardenas. 2011. Head-enders as stationary bandits in asymmetric commons: Comparing irrigation experiments in the laboratory and the field. Ecological Economics 70(9):1590-1598.

Keen, M., V. A. Brown, and R. Dyball. 2005. Social Learning in Environmental Management: Towards a Sustainable Future. Earthscan, New York, NY.

Newig, J., D. Gunther, and C. Pahl-wostl. 2010. Synapses in the Network : Learning in Governance Networks in the. Ecology and Society 15(4):24.

Olsson, P., C. Folke, and F. Berkes. 2004. Adaptive comanagement for building resilience in social-ecological systems. Environmental management 34(1):75-90.

Ostrom, E. 1990. Governing the commons: The evolution of institutions for collective action. Cambridge University Press, Cambridge, UK.

Ostrom, E. 1992. Crafting institutions for self-governing irrigation systems. ICS Press, San Francisco, CA.

Ostrom, E., and R. Gardner. 1993. Coping with Asymmetries in the Commons : Self-Governing Irrigation Systems Can Work. The Journal of Economic Perspectives 7(4):93-112. 
Pahl-Wostl, C. 2009. A conceptual framework for analysing adaptive capacity and multi-level learning processes in resource governance regimes. Global Environmental Change 19(3):354-365.

Park, J., T. P. Seager, and P. S. C. Rao. 2011. Lessons in risk- versus resilience-based design and management. Integrated Environmental Assessment and Management 7(3):396-399.

Pavitt, C. 2011. Communication, Performance, and Perceptions in Experimental Simulations of Resource Dilemmas. Small Group Research 42(3):283-308.

Perez, I., D. J. Yu, M. A. Janssen, and J. M. Anderies. 2015. Social roles and performance of social-ecological systems: evidence from behavioral lab experiments. Ecology and Society 20(3):23.

Plummer, R., and D. Armitage. 2007. A resilience-based framework for evaluating adaptive comanagement: Linking ecology, economics and society in a complex world. Ecological Economics 61(1):62-74.

Polasky, S., S. R. Carpenter, C. Folke, and B. Keeler. 2011. Decision-making under great uncertainty: Environmental management in an era of global change. Trends in Ecology and Evolution 26(8):398-404.

Poteete, A. R., E. Ostrom, and M. A. Janssen. 2010. Working Together: Collective Action, the Commons, and Multiple Methods in Practice. Princeton University Press, Princeton, New Jersey, USA.

Ragin, C. C. 1987. The Comparative Method - Moving Beyond Qualitative and Quantitative Strategies. Encyclopedia of Social Science Research Methods. University of California Press, Berkeley, CA.

Ragin, C. C. 2000. Fuzzy-Set Social Science. University of Chicago Press, Chicago, IL.

Ragin, C. C. 2008. User's Guide to Fuzzy-Set/Qualitative comparative Analysis 2.0. Department of Sociology, University of Arizona, Tucson, AZ.

Reed, M. S., A. C. Evely, G. Cundill, I. Fazey, J. Glass, A. Laing, J. Newig, B. Parrish, C. Prell, C. Raymond, and L. C. Stringer. 2010. What is social learning? Ecology and Society 15(4):1.

Rodela, R. 2011. Social Learning and Natural Resource Management: The Emergence of Three Research Perspectives. Ecology and Society 16(4):30.

Russell, D. B., and R. L. Ison. 2000. The research-development relationship in rural communities: an opportunity for contextual science. Pages 10-31 in D. B. Russell and R. L. Ison, editors. Agricultural Extension and Rural Development: Breaking out of Traditions. Cambridge University Press, Cambridge, UK.

Stiglitz, J. E., and B. C. Greenwald. 2014. Creating a Learning Society: A New Approach to Growth, Development, and Social Progress (Kenneth J. Arrow Lecture Series). Columbia University Press, New York, NY.

Walker, B., C. S. Holling, S. R. Carpenter, and A. Kinzig. 2004. Resilience, Adaptability and Transformability in Social-ecological Systems. Ecology And Society 9(2):5.

Walters, C. J., and C. S. Holling. 1990. Large-Scale Management Experiments and Learning by Doing. Ecology 71(6):2060-2068. 